\title{
Alternate Paths for Multiple Fault Tolerance on Dynamic WDM Optical Networks
}

\author{
Nicolas Jara \\ UTFSM \\ Valparaíso, Chile \\ and INRIA Rennes - Bretagne Atlantique \\ Rennes, France \\ Email: nicolas.jara@usm.cl
}

\author{
Gerardo Rubino \\ INRIA Rennes - Bretagne Atlantique \\ Rennes, France \\ Email: gerardo.rubino@inria.fr
}

\author{
Reinaldo Vallejos \\ UTFSM \\ Valparaíso, Chile \\ Email: reinaldo.vallejos@usm.cl
}

\begin{abstract}
This paper proposes a new method to compute alternate routes for multiple fault tolerance on Dynamic WDM Optical Networks. The method allows to obtain all the paths that replace the primary routes affected by one or several failures. Additional paths, called secondary routes, are used to keep each user connected to the network, including cases where multiple simultaneous link failures occur. The method also allows to obtain the number of wavelengths in each link of the network, computed such that the blocking probability of each connection is less than a pre-defined threshold (which is a network design parameter), in spite of the occurrence of $k$ simultaneous link failures, with $k \geq 1$.

The solution obtained by the new algorithm is significantly more efficient than the result of applying current methods, its implementation is notably simple and its on-line operation is very fast.
\end{abstract}

Index Terms-Dynamic WDM optical networks, Routing, Wavelength Dimensioning, Multiple Fault Tolerance

\section{INTRODUCTION}

The rapid increase on demand for bandwidth from existing networks has caused a growth in the use of technologies based on WDM optical networks. This technology can transmit data over multiple channels on a single fiber, reaching transmission rates of Tb/s per fiber [1]. Currently, this type of network is operated statically [1], i.e., the route assigned to each user (connection) is permanently assigned from source to destination, regardless of the percentage of time it is used. This type of operation is inefficient in the usage of network resources, especially for low traffic loads which is the most common case.

One way to help overcome the inefficiencies of static networks is to migrate them to a dynamic operation. A dynamic operation consists in allocating the resources required by each user only when it has data to transmit. A possible lack of resources to successfully transmit a burst can happen because dynamic networks are designed based on a statistical commitment: on one hand, to save costs, they are designed with the less possible amount of resources; on the other hand, to be efficient, they are designed to avoid the occurrence of burst losses (blocking). To achieve a balance between these two opposite features, the network must be designed such that for any end-to-end connection, the blocking probability is less than or equal to a design parameter $B$, typically pretty close to 0 .

One of the main problems that need to be solved in order to design dynamic WDM networks is to define the route to be used by each possible connection to transmit its information. This problem is known as Routing. It must also be determined how many wavelengths should be assigned to each link of the network, in order to achieve the compromise between efficiency and cost previously described. This problem is called Wavelengths Dimensioning. Another issue to be solved is to ensure that the network will still be able to provide its transmission service after the failure of one or more of its links. The solution to this problem consists in providing the necessary infrastructure to rapidly re-establish communications between all source-destination pair of nodes affected by these link failures. This type of mechanism is known as Fault Tolerance.

The frequency of link failure occurrence is significant. For instance, [2], [3] report measures of the mean time between failures of about 367 [Year $/ \mathrm{km}$ ]. This means that failures on links significantly impact the performance of networks. For example, in a 26,000 km-long network as NSFNet [4] (Figure 1), there is an average of one fiber cut every 5 days. Moreover, it has been found that the frequency with which two simultaneous network failures occur is high enough to be considered in the network design. For example, in [3] it has been reported that the probability of two simultaneous failures occurring in a network like NSFNet is approximately 0.0027 (this corresponds to a downtime of about 24 hours per year on average), which in addition to the high transmission rate of this kind of transmission infrastructure, implies an unacceptable loss for a network operator.

The remainder of this paper is as follows: Section II describes the state of the art related to Routing, Wavelength Dimensioning, and Fault Tolerance Capacity on Dynamic WDM Optical Networks; Section III presents our new algorithm; Section IV contains the results obtained for different network topologies by the proposed algorithm, and are contrasted with those obtained with the method normally used in the literature for this purpose. Finally, the conclusions of 
the work are given in Section V.

\section{STATE OF THE ART}

The fault tolerance methods proposed so far generally have been devoted to finding alternative paths considering single link failure. Then, the number of wavelengths in the network is dimensioned to tolerate this situation [3], [5], [6]. However, as already noted, the probability of occurrence of two or more simultaneous failures is often high enough, making that it is useful to consider this event in the design of the network. Some studies have focused on this scenario [7]-[10], which are described next.

1+1 Method: In this method, for each primary route a secondary route is assigned, disjoint with the former (meaning that they don't share any link), and the information is transmitted simultaneously through both routes [11], [12]. We denoted this method as Fault Tolerance Method Based on $1+1(F T B 1+1)$. To dimension the number of wavelengths of each link (task usually done by simulation), each secondary route is considered as just another network route with a load equal to the corresponding primary route. The FTB $1+1$ method is scalable to provide tolerance to $k$ simultaneous failures (with $k \geq 1$ ). In this case, for each connection $k+1$ disjoint routes must be found, one is the primary route and the other $k$ are secondary routes.

P-cycle Method: In [8], [13] a method of fault tolerance named P-cycle is used, which allows sharing resources through fixed secondary routes, which have a cyclic form. These routes are shared between several primary routes. One problem with this approach is that the applicability of p-cycles is very dependent on the size of the network, introducing excessive additional delay for a connection in protection state on large networks. Also, to perform multiple failure restorability, this method requires a great amount of cycles (e.g. hundreds of cycles of the 11 nodes pan-European COST 239 network [13]), which is impractical from various points of view.

Shared Path Protection Method: Another strategy was described in [10] in order to provide fault tolerance to double link failures. In this method the extra resources (wavelengths) assigned to the secondary routes can be shared by different connections, and are assigned only when a fault occurs. This method is known as "Shared Path Protection" [14], [15]. It can be executed in two different ways. The first is running the algorithm off-line, where the routes are calculated prior to the operation of the network (SPP offline). The second way is the on-line implementation (SPP online). In this last case, the method is executed every time there is a change in the network (link failure or traffic load variation). In the SPP online mode, the primary routes are specified before the network is operating, but in order to find new routes to the affected connections it must be executed again every time that one or more simultaneous failures occur, therefore, it is a proactive and a reactive approach at the same time.

\section{THE PROPOSED METHOD}

The network topology is represented by a graph $\mathcal{G}=$ $(\mathcal{V}, \mathcal{L})$, where $\mathcal{V}$ is the set of network nodes or vertices and $\mathcal{L}$ is the set of unidirectional links (the arcs in $\mathcal{G}$ ), with respective cardinalities $|\mathcal{V}|=V$ and $|\mathcal{L}|=L$. The set of connections $\mathcal{X} \subseteq \mathcal{V}^{2}$, with cardinality $|\mathcal{X}|=X$, is composed by all the source-destination pairs with communication between them. These connections are also called "users" in the text.

To represent the traffic between a given source-destination pair, an ON-OFF model is used. Consider connection $c$. During any of its ON periods, whose average length is $t_{O N}$, the source transmits at a constant rate. During an OFF period, with average length $t_{O F F}$, the source refrains from transmitting data.

When traffic sources are ON, they all transmit at the same rate, determined by the used technology, that to simplify the presentation will be our rate unity. Consequently, the traffic load of connection $c$, denoted by $\varrho_{c}$, is given by the following expression:

$$
\varrho=\frac{t_{O N}}{t_{O N}+t_{O F F}} .
$$

Let $\mathcal{R}=\left\{r_{c} \mid c \in \mathcal{X}\right\}$ be the set of routes (primary routes) that enable communications among the different users, where $r_{c}$ is the route associated with connection $c \in$ $\mathcal{X}$. The set of connections passing through link $\ell$ in the graph is denoted by $\mathcal{T}_{\ell}$, and we denote by $N_{l}$ their number, that is, $N_{\ell}=\left|\mathcal{T}_{\ell}\right|$.

Let $\mathcal{W}=\left\{W_{l} \mid \ell \in \mathcal{L}\right\}$ be the set containing the number of wavelengths associated with each unidirectional network link, where $W_{l}, \ell \in \mathcal{L}$, is the number of wavelengths on link $\ell$. The value $W_{l}$, for every $\ell \in \mathcal{L}$, will be evaluated so that the blocking probability of each connection $c \in \mathcal{X}$ is less than or equal to a given pre-specified threshold and the total number of available network wavelengths is as small as possible.

It is assumed that before running the fault tolerance method, the primary routes and their dimensioning (that is, sets $\mathcal{R}$ and $\mathcal{W}$ ) have been already obtained using any method available.

As in [16], [17], in this work the total network cost $C_{\text {net }}$ is defined as the sum of all wavelengths of all network links, but as we are considering fault tolerance capabilities, this includes all the extra wavelengths needed to provide tolerance to multiple link failures. This definition is based on the fact that the cost of many of the components of an optical network is strongly affected by this parameter. The parameter $C_{n e t \_n f t}$ is defined as the number of wavelengths required to solely provide connection to the primary routes (this means without considering fault tolerance), that is, $C_{n e t_{-} n f t}=\sum_{l: l \in \mathcal{L}} W_{l}$. Later, $C_{n e t_{-} e f t}$ is defined as the additional number of wavelengths needed by the network to provide single (or multiple) fault tolerance, i.e. $C_{n e t_{-} e f t}=$ $C_{n e t}-C_{n e t \_n f t}$.

Additionally the following definitions concerning random objects will be used. Consider a set of possible failure scenarios $\Omega$ on the network. Each of these scenarios is 
a subset of failed network links $\mathcal{F}$, where $\mathcal{F} \subset \mathcal{L}$ with cardinality $|\mathcal{F}| \leq K$. This subset $\mathcal{F}$ corresponds to a failure scenario, where every links belonging to $\mathcal{F}$ is non operational (failed). Therefore, this method can be applied to any possible case of failures in the network, e.g. every possible single failure scenario $(|\mathcal{F}|=1)$, every possible double failure scenario $(|\mathcal{F}|=2$ ), node failure (corresponding to a special case of multiple failure, where all the links connected to that node are non operational), etc. We can also handle disaster risk constrains [18] and Shared-RiskGroup constrains scenarios [19]. Disaster risk constrains considers the possible service disruptions in case of a natural disaster or a targeted attack. This scenario can be considered in our method by including in the failure scenario $\mathcal{F}$ all the links where the disaster has taken place. On the other hand, Shared-Risk-Group (SRG) considers the possibility that some fibers are placed physically together, even if they are connecting different optical nodes. This scenario makes them all liable to physical cuts, since they can be cut together at the same time. This case can be considered also as a special case of multiple link failures, where $\mathcal{F}$ is composed by every link that can be affected by the same physical cut.

- $\mathcal{G}^{-\mathcal{F}}=(\mathcal{N}, \mathcal{L} \backslash \mathcal{F})$, is the partial graph of $\mathcal{G}$ (same nodes, part of the edges), containing only the non-failed links.

- $\mathcal{X}^{\mathcal{F}}=\left\{c \mid \mathcal{F} \in r_{c}\right\}$, is the set of all network connections whose routes pass through any of the failed links.

- $\mathcal{A}^{\mathcal{F}}$, is the set of routes that are affected when all the links in $\mathcal{F}$ fail.

- $\mathcal{R}^{\mathcal{F}}$, is the set of secondary routes associated with all connections $c \in \mathcal{X}^{\mathcal{F}}$.

Using this notation, next we describe the method proposed in this paper, called "Fault Tolerance Method Based on Cheapest Paths" (FTBCP). We explain first the algorithm as a set of steps, and then a pseudo-code is presented considering each step in an algorithm.

Notice that, this procedure must be repeated for each possible failure scenario in $\Omega$.

Step 1: Arbitrarily select a set of links $\mathcal{F}=\{l \mid l \in \mathcal{L}\}$ which are considered failed, where the number of elements of $\mathcal{F}$ is $k$.

Step 2: With each working link $l \in \mathcal{L} \backslash \mathcal{F}$, we associate the $\operatorname{cost} C_{l}^{-\mathcal{F}}=W_{l} / N_{l}$, where the variable $N_{l}$ is the number of users using the link $l$ and $W_{l}$ is the amount of resources needed to attend with the quality of service required to such $N_{l}$ users. This dimensioning step may be solved using the method published on [20]. The subjacent idea behind this cost function is that $W_{l} / N_{l}$ is the amount of resources needed by the system to serve 1 user with the quality of service required. Consequently, $\sum_{\forall l \in r_{c}} W_{l} / N_{l}$ is equal to the network cost required to serve the user $c$, end to end. Then, the cheapest end to end routes are established using the Dijkstra's algorithm, where $W_{l} / N_{l}$ is used to represent the cost of the 1-th link, for each of the network links.
Step 3: Using the Dijkstra algorithm, calculate the cheapest route of each connection $c$ affected by the failure of the set of links $\mathcal{F}$, where each route cost is evaluated as the sum of the cost associated with its links. Each of these routes are secondary routes which are stored in a set called $\mathcal{R}^{\mathcal{F}}$. Next, compute $\mathcal{S}^{\mathcal{F}}$, which contains all primary routes except those affected by the failures of the links in $\mathcal{F}$, plus the secondary routes of the affected connections. In symbols, $\mathcal{S}^{\mathcal{F}}=\left(\mathcal{R} \backslash \mathcal{A}^{\mathcal{F}}\right) \cup \mathcal{R}^{\mathcal{F}}$.

Step 4: Using the $\mathcal{S}^{\mathcal{F}}$ set on $\mathcal{G}^{-\mathcal{F}}$ graph, calculate: the number of $N_{l}^{\mathcal{F}}$ connections passing through each link $l \in \mathcal{L}^{-\mathcal{F}}$; the number of wavelengths $W_{l}^{\mathcal{F}}$ necessary to each link $l \in \mathcal{L}^{-\mathcal{F}} ;$ and the total network cost $C_{\text {net }}^{\mathcal{F}}=\sum_{l: l \in \mathcal{L}^{-\mathcal{F}}} W_{l}^{\mathcal{F}}$, where $W_{l}^{\mathcal{F}}$ is calculated so that the maximum acceptable blocking probability $B$ is achieved, for which simulation or an existing mathematical method from literature can be used i.e. [20].

Observation: Steps 2 to 5 are repeated, but starting with the routes and number of wavelengths calculated in the previous steps, forming the iterative part of FTBCP method. This steps must be repeated at least 1 time.

Step 5: Consider $C_{\text {net2 }}^{\mathcal{F}}$ and $C_{\text {net }}^{\mathcal{F}}$ as the netowrk cost calculated on step 4 in the actual and previous iteration respectively. If $C_{\text {net } 2}^{\mathcal{F}}<C_{\text {net }}^{\mathcal{F}}$, make $C_{\text {net }}^{\mathcal{F}}=C_{\text {net } 2}^{\mathcal{F}}$, and save it as the best result obtained to this point, then steps 2 to 5 are repeated. The repetition of steps 2 to 5 stops when the $\operatorname{cost} C_{n e t}^{\mathcal{F}}$ converges to a fixed value. Our convergence test is here simply the fact that the obtained cost doesn't change from an iteration to the next one. These repetitions allow to find the lowest possible network cost within the philosophy of this algorithm. After reaching convergence, the secondary routes $\mathcal{R}^{\mathcal{F}}$ and the dimensioning $\left(W_{l}^{\mathcal{F}}\right)$ obtained are stored for later use.

Step 6: For each different set of network links $\mathcal{F}$, such that $\mathcal{F}$ contains $k$ elements, repeat step 1 to 6 .

Step 7: The final dimensioning of each network link $l$ is equal to the maximum obtained in the previous steps, i.e. $W_{l}^{\text {final }}=\max _{\mathcal{F}}\left(W_{l}^{\mathcal{F}}\right)$. This means that the network is dimensioned to withstand the worst possible failure scenario. It should be remarked that in this way each link is dimensioned without any distinction between primary and secondary routes, also considering each possible case of link failure.

Step 8: The set $\mathcal{D}=\left\{W_{l}^{\text {final }}, l \in \mathcal{L}\right\}$ is stored as the set containing the number of wavelengths for each network link $l$. Next, for each $\mathcal{F}$, such that $|\mathcal{F}|=k$, the sets $\mathcal{F}$ and $\mathcal{R}^{\mathcal{F}}$ which contains all secondary routes associated with each possible link failures $\mathcal{F}$ are stored.

Note that the FTBCP method allows to provide fault tolerance to multiple simultaneous connections, as long as the network topology remains connected despite the failure of $k$ links.

Note that the FTBCP algorithm must be executed off-line for the maximum traffic load expected. As a consequence, the set of routes are stored in routing tables. Then, in the 
on-line operation of the network, all the route information for each connection (both primary and secondary) can be obtained on demand. This ensures a very fast network online operation, in case of a failure.

The corresponding pseudo-code of the entire strategy is summarized in Algorithm 1. In this pseudo-code two functions are used.

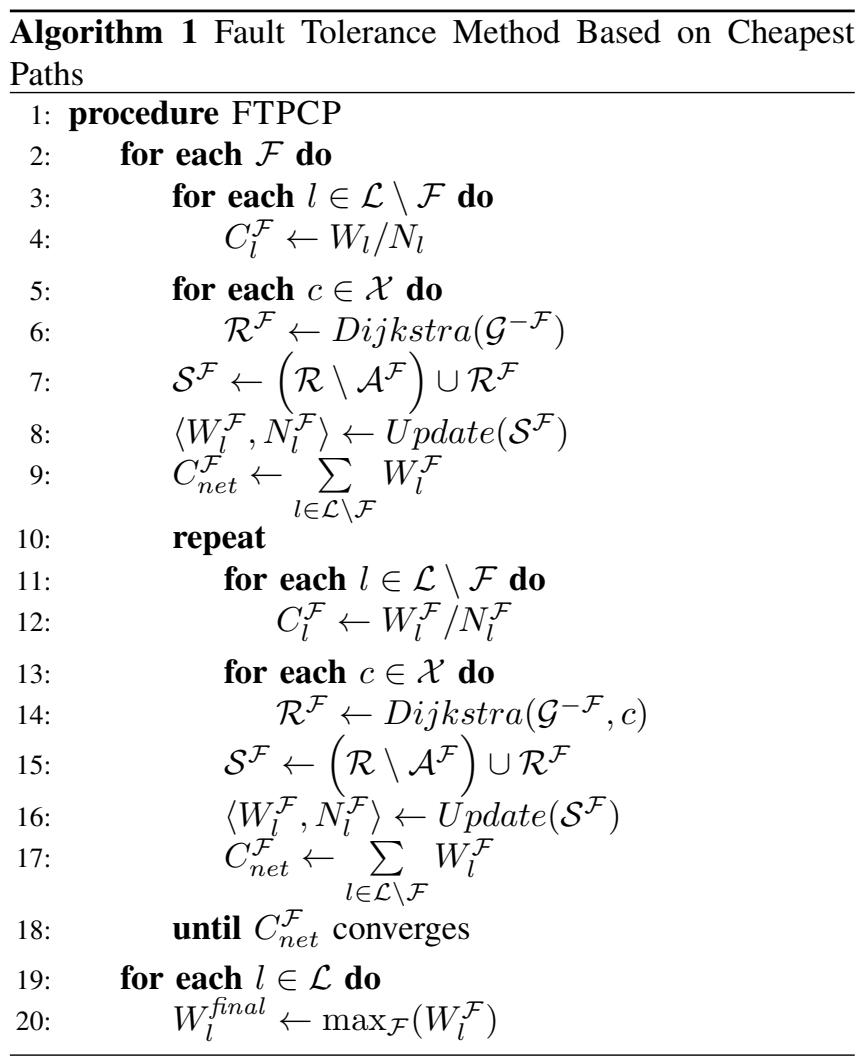

- $\operatorname{Dijkstra}\left(\mathcal{G}^{-\mathcal{F}}, c\right)$ : This function calculates the cheapest route for the connection $c$, considering the network graph $\mathcal{G}^{-\mathcal{F}}$ with the updated link costs $C_{l}^{\mathcal{F}}$.

- $\operatorname{Update}\left(\mathcal{S}^{\mathcal{F}}\right)$ : For a given set of routes $\mathcal{S}^{\mathcal{F}}$, this function evaluates the number of users passing through each link $l \in \mathcal{L}$, and the number of wavelengths required on each link $l \in \mathcal{L}$ guaranteeing a predefined quality of service (each connection blocking probability), using the algorithm proposed on [20].

\section{NumericAl Results}

To quantify the quality of the solution obtained by the FTBCP method, it should be compared against the optimal solution. However, it is known that the R\&WD problem is an NP-complete problem [21]. Those who have been dedicated to solve this problem optimally only have been able to achieve it to very small networks (with less than 10 nodes). Consequently, for real network topologies (number of nodes of some tens) fault tolerance cannot be solved optimally. Given this situation, our best alternative was to compare the FTBCP method with those methods considered as the most competitive at this moment.
Then, it is necessary to make the comparison with respect to metrics that enable to assess the advantages/disadvantages of each fault tolerance method. The most important metrics for the routing, dimensioning and fault tolerance methods are: the cost of the network and the delay in the restoration procedure in case of the occurrence of failures, when this is relevant (in our approach this delay is negligible since the computations are done off-line).

a) Shared Path Protection (SPP) Method.: There are two methods for implementing this algorithm (SPP online and SPP off-line). Both methods require between 40 to $80 \%$ of additional wavelengths (compared to the case without fault tolerance) to provide single link fault tolerance capability [14]. On the SPP off-line method the percentage of restorability obtained (percentage of connections that remain connected in case of link failure) is very low (80\% to $90 \%$ [14]). Therefore, it is not comparable to the method proposed in this work, which garantees a QoS defined by the network designer (i.e. $10^{-6}$ ). The SPP-online method requires to run on demand a route search algorithm (whenever one or more links fail) in order to find an alternative route to each affected connection. Evidently, this on-line strategy causes a slow re-routing. Then, this type of method does not represent a practical fault tolerant mechanism for many practical applications. Therefore, the SPP method was not considered for comparison with the method proposed in this paper.

b) The p-cycle Method. : To provide tolerance to multiple failures, this method requires a large quantity of cycles (which implies a high cost when defining secondary routes), so it is not scalable for multiple faults. Given the fact that in this paper we consider the multiple fault tolerant case, it is unreasonable to compare our method with the $p$ cycle one.

c) Method 1+1.: This method provides tolerance to multiple failures, using as many disjoint routes as simultaneous link failures it can tolerate. It solves the problem of primary and secondary routes prior to the network dimensioning (off-line) sub-task. Then, the number of wavelengths is computed, having as a constraint to provide enough resources to all routes, and guaranteeing sufficient information to re-route each connection in case of failure. Consequently, $1+1$ is the most suitable fault tolerance method to compare with our algorithm.

To solely compare the effect of the fault tolerance algorithm, the same set of primary routes $\mathcal{R}$ (which were generated by the SPBR method [22]), and the same wavelength dimensioning method (proposed in [20]) were used. Therefore, hereinafter, only the secondary routes are unique to each method, therefore we compare the additional network costs needed to tolerate simple (or multiple) fault tolerance, denoted as $C_{n e t \_e f t}$.

To evaluate the performance of the proposed method under different scenarios, the algorithm was executed for real network topologies of different sizes and different degrees of connection $d$, where $d$ is the average number of neighbors of a node, as shown on Figure 1. 

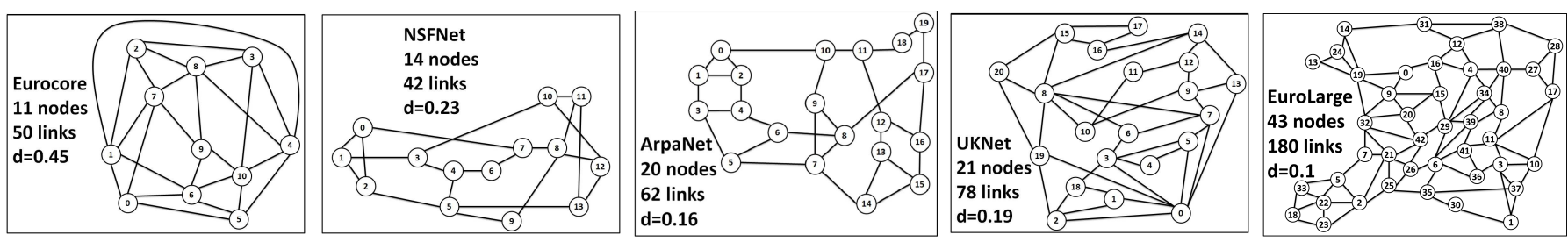

Figure 1. Mesh networks evaluated. Each edge on the networks are bidirectional, so the number of links $L$ refers to unidirectional arcs on each graph. The parameter $d$ is a measure of density: if the graph has $L$ arcs and $N$ nodes, then $d=L /(N(N-1))$.
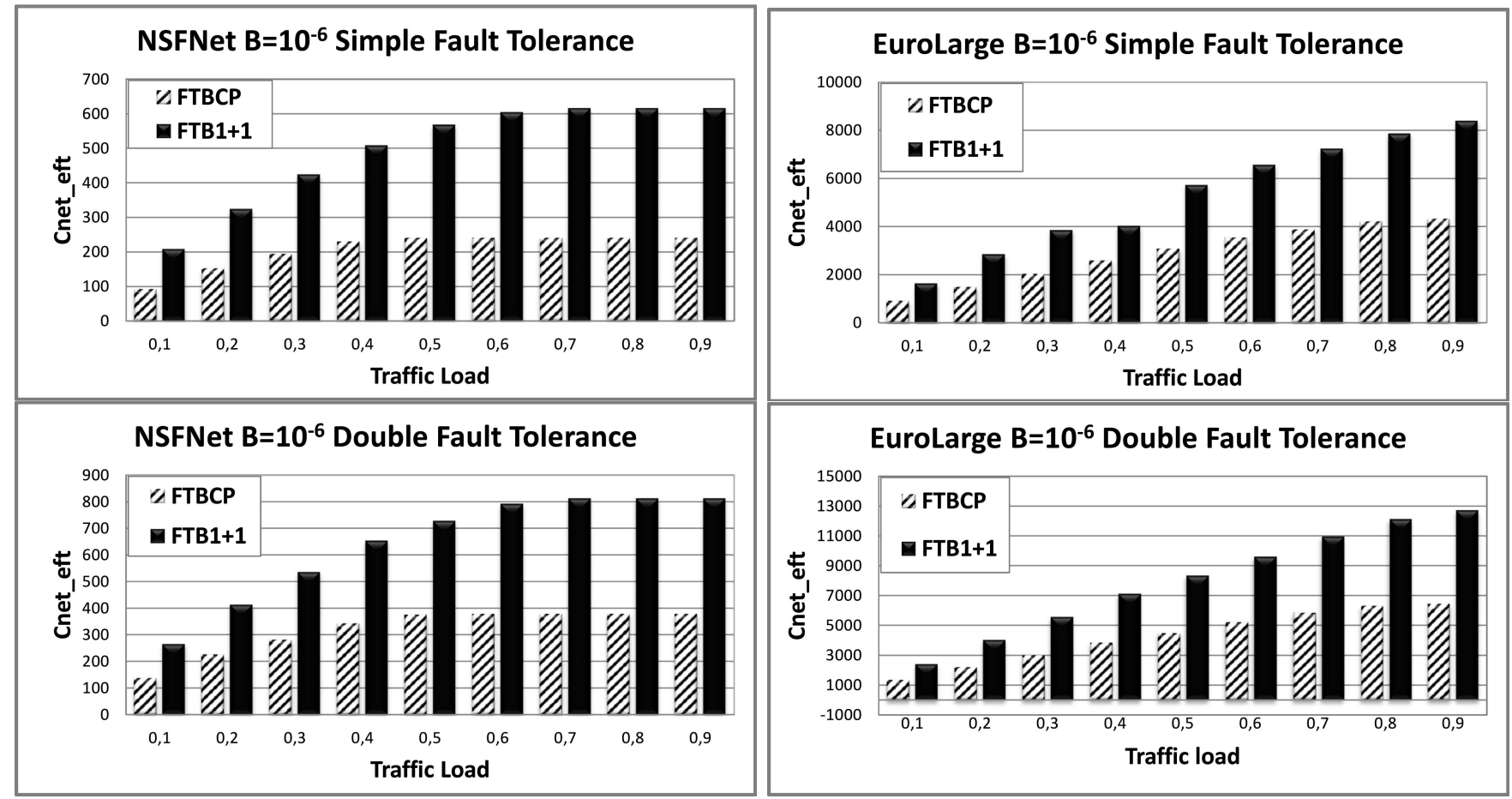

Figure 2. The extra number of wavelengths required, $C_{n e t_{-} \text {eft }}$, obtained with FTBCP and FTB1+1, considering the case of simple and simultaneous double failure for the network topologies NSFNet and EuroLarge, as a function of the traffic load and for a maximum acceptable blocking probability per connection of $10^{-6}$.

Results: Figure 2 shows the values of $C_{\text {net_eft }}$ obtained by the FTBCP and FTB1+1 methods, as a function of the traffic load, for a maximum acceptable blocking probability per connection of $10^{-6}$, in the case of simple failure (upper charts) and simultaneous double failure (lower charts) and for different network topologies.

As it can be seen in Figure 2, in the case of tolerance to a single failure, the FTBCP method performs clearly better. In fact, for all the scenarios evaluated the FTB1+1 method requires in the order of $50 \%$ more wavelengths (for $\varrho=0,3$ which represents a typical network load [1]) than the cost of the method proposed herein. In the case of tolerance to two simultaneous failures of links, the FTBCP method also significantly outperforms the FTB $1+1$ technique. In this last case, the FTB $1+1$ method requires in the order of $60 \%$ more wavelengths (for $\varrho=0,3$ [1]) than our proposal.

To better exemplify such difference, in Table I, the total cost $C_{n e t}$ is shown, measured in number of wavelengths for the following cases: without fault tolerance; single link failure; and simultaneous double fault link: This for the same network topologies as in the Figure 1, and considering a maximum blocking probability of $10^{-6}$ for a traffic load of 0.3 . Notice that the first the results of $C_{n e t \_n f t}$ are the same for both methods, since they are using the same primary routes as we previously explained.

\section{CONCLusions}

A new method was proposed to solve the problem of tolerance to multiple link failures in Dynamic WDM Optical Networks with full Wavelength Conversion. The method is applicable to any of the algorithms currently used to define the set $\mathcal{R}$ of primary routes.

The proposed methodology differs considerably from those published so far. The main differences are: a) It jointly defines the set of secondary routes, and determines the number of wavelengths on each network link so that the blocking probability of any user is lower than a certain pre-specified threshold (which is a design parameter of the 


\begin{tabular}{|c|c|c|c|c|c|c|c|}
\hline \multicolumn{2}{|c|}{ Parameters } & \multicolumn{2}{c|}{$C_{\text {net_nft }}$} & \multicolumn{2}{c|}{$C_{n e t}$ Simple FT } & \multicolumn{2}{c|}{$C_{n e t}$ Double FT } \\
\hline Topology & $\varrho$ & FTBCP & FTB1+1 & FTBCP & FT1+1 & FTBCP & FT1+1 \\
\hline Eurocore & 0.3 & 174 & 174 & 270 & 398 & 321 & 454 \\
NSFNet & 0.3 & 390 & 390 & 584 & 814 & 671 & 926 \\
ArpaNet & 0.3 & 922 & 922 & 1381 & 1845 & 1584 & 2221 \\
UKNet & 0.3 & 974 & 974 & 1449 & 1878 & 1621 & 2242 \\
EuroLarge & 0.3 & 4570 & 4570 & 6604 & 8424 & 7570 & 10144 \\
\hline
\end{tabular}

Table I

TOTAL NUMBER OF WAVELENGTHS REQUIRED FOR THE METHOD FTBCP AND FTB $1+1$ FOR CASES: NO FAULT TOLERANCE $\left(C_{n e t} n f t\right)$, SIMPLE Fault $\left(\left(C_{n e t}\right.\right.$ Simple FT), and Simultaneous double fault $\left(C_{n e t}\right.$ Double FT), For Eurocore, NSFNET, ARPANET, UKNET and EUROLARGE NETWORKS, CONSIDERING A MAXIMUM BLOCKING PROBABILITY OF $10^{-6}$ AND A LOAD OF TRAFFIC 0.3.

network). b) By simultaneously solving both problems, a global solution is obtained, which is more efficient than a solution obtained by solving each problem separately. In fact, it is more efficient than current methods in terms of number of wavelengths (the current method is associated with a $50 \%$ higher cost for single faults, at least for the typical topologies that were analyzed, and the gain is higher in the case of tolerance to double faults). c) It dimensions the number of wavelengths without making any distinction between primary and secondary routes; therefore, it better exploits statistical multiplexing among all connection requests. d) It is scalable to more than two simultaneous faults, as long as the network topology allows reconnection via the links that remain operating. e) The proposed method is executed before the network is operating (off-line); in this way, the on-line operation of the network is simple and fast, since the routes (both primary and secondary) are stored in routing tables which are consulted under demand. f) It requires a few seconds to do the routing and the wavelength dimensioning; as a consequence, the procedure also could be used in an on-line version, for example, to deal with traffic load variations during the network operation.

\section{ACKNOWLEDGMENTS}

This work received financial support from FONDEF ID14I10129, Stic/Amsud-Conicyt project “DAT", USM PIIC Proyect, CONICYT $\mathrm{PhD}$ and CONICYT PhD internship scholarships. These projects and institutions are then gratefully acknowledged.

\section{REFERENCES}

[1] A. A. M. Saleh and J. M. Simmons, "Technology and architecture to enable the explosive growth of the internet," Communications Magazine, IEEE, vol. 49, pp. 126-132, January 2011.

[2] M. To and P. Neusy, "Unavailability analysis of long-haul networks," Selected Areas in Communications, IEEE Journal on, vol. 12, pp. 100-109, Jan 1994.

[3] D. A. Schupke, A. Autenrieth, and T. Fischer, "Survivability of multiple fiber duct failures," in Third International Workshop on the Design of Reliable Communication Networks (DRCN, pp. 7-10, 2001.

[4] R. Ramaswami and K. Sivarajan, "Design of logical topologies for wavelength-routed all-optical networks," in INFOCOM '95. Fourteenth Annual Joint Conference of the IEEE Computer and Communications Societies. Bringing Information to People. Proceedings. IEEE, pp. 1316-1325 vol.3, Apr 1995.

[5] H. Singh, J. Prakash, D. Arora, and A. Wason, "Fault tolerant congestion based algorithms in OBS network," International Journal of Engineering (IJE), vol. 5, no. 5, 2011.
[6] F. S. H. Souza, D. L. Guidoni, and G. R. Mateus, "A column generation-based heuristic for the grwa with protection and qos in wdm optical networks," in Computers and Communications (ISCC), 2013 IEEE Symposium on, pp. 000922-000927, July 2013.

[7] D. Mukherjee, C. Assi, and A. Agarwal, "Alternate strategies for dual failure restoration using p-cycles," in Communications, 2006. ICC '06. IEEE International Conference on, vol. 6, pp. 2477-2482, June 2006.

[8] R. Yadav, R. Yadav, and H. Singh, "Intercycle switching (ics)-based dynamic reconfiguration of p-cycle for dual-failure survivability of wdm networks," Photonic Network Communications, vol. 24, no. 2, pp. 160-165, 2012.

[9] L. Tang, M. Cai, B. Li, and R. Wu, "A novel multi-link faulttolerant algorithm for survivability in multi-domain optical networks," Photonic Network Communications, vol. 24, no. 2, pp. 77-85, 2012.

[10] D. S. Yadav, S. Rana, and S. Prakash, "A mixed connection recovery strategy for surviving dual link failure in $\{\mathrm{WDM}\}$ networks," Optical Fiber Technology, vol. 19, no. 2, pp. $154-161,2013$.

[11] S. Chalasani and V. Rajaravivarma, "Survivability in optical networks," in System Theory, 2003. Proceedings of the 35th Southeastern Symposium on, pp. 6-10, March 2003.

[12] P. C. Srikanth, M. V. Sathyanarayana, D. Balaraju, and T. Srinivas, "Fault tolerance in survivable wdm optical networks using hybrid scheme: An analytical approach," in TENCON 2009 - 2009 IEEE Region 10 Conference, pp. 1-6, Jan 2009.

[13] D. Schupke, "Multiple failure survivability in wdm networks with pcycles," in Circuits and Systems, 2003. ISCAS '03. Proceedings of the 2003 International Symposium on, vol. 3, pp. III-866-III-869 vol.3, May 2003.

[14] D. Schupke and R. Prinz, "Capacity efficiency and restorability of path protection and rerouting in wdm networks subject to dual failures," Photonic Network Communications, vol. 8, no. 2, pp. 191-207, 2004.

[15] A. Wason and R. Kaler, "Fault-tolerant routing and wavelength assignment algorithm for multiple link failures in wavelength-routed all-optical $\{$ WDM $\}$ networks," Optik - International Journal for Light and Electron Optics, vol. 122, no. 2, pp. 110 - 113, 2011.

[16] R. Ramaswami, K. Sivarajan, and G. Sasaki, Optical Networks: A Practical Perspective, 3rd Edition. San Francisco, CA, USA: Morgan Kaufmann Publishers Inc., 3rd ed., 2009.

[17] R. Vallejos and N. Jara, "Join routing and dimensioning heuristic for dynamic $\{W D M\}$ optical mesh networks with wavelength conversion," Optical Fiber Technology, vol. 20, no. 3, pp. 217 - 223, 2014.

[18] S. Ferdousi, F. Dikhiyik, M. F. Habib, and B. Mukherjee, "Disasteraware data-center and content placement in cloud networks," in 2013 IEEE International Conference on Advanced Networks and Telecommunications Systems (ANTS), pp. 1-3, IEEE, 2013.

[19] H. Zang, C. Ou, and B. Mukherjee, "Path-protection routing and wavelength assignment in wdm mesh networks under shared-risk-group constraints," in Asia-Pacific Optical and Wireless Communications Conference and Exhibit, pp. 49-60, International Society for Optics and Photonics, 2001.

[20] R. Vallejos, A. Zapata, and M. Aravena, "Fast and effective dimensioning algorithm for end-to-end optical burst switching networks with ON-OFF traffic model," in Optical Network Design and Modeling, pp. 378-387, Springer, 2007.

[21] R. Vallejos, A. Zapata-Beghelli, V. Albornoz, and M. Tarifeño, "Joint routing and dimensioning of optical burst switching networks," Photonic Network Communications, vol. 17, no. 3, pp. 266-276, 2009.

[22] J. Teng and G. Rouskas, "Traffic engineering approach to path selection in optical burst switching networks," J. Opt. Netw., vol. 4, pp. 759-777, Nov 2005. 